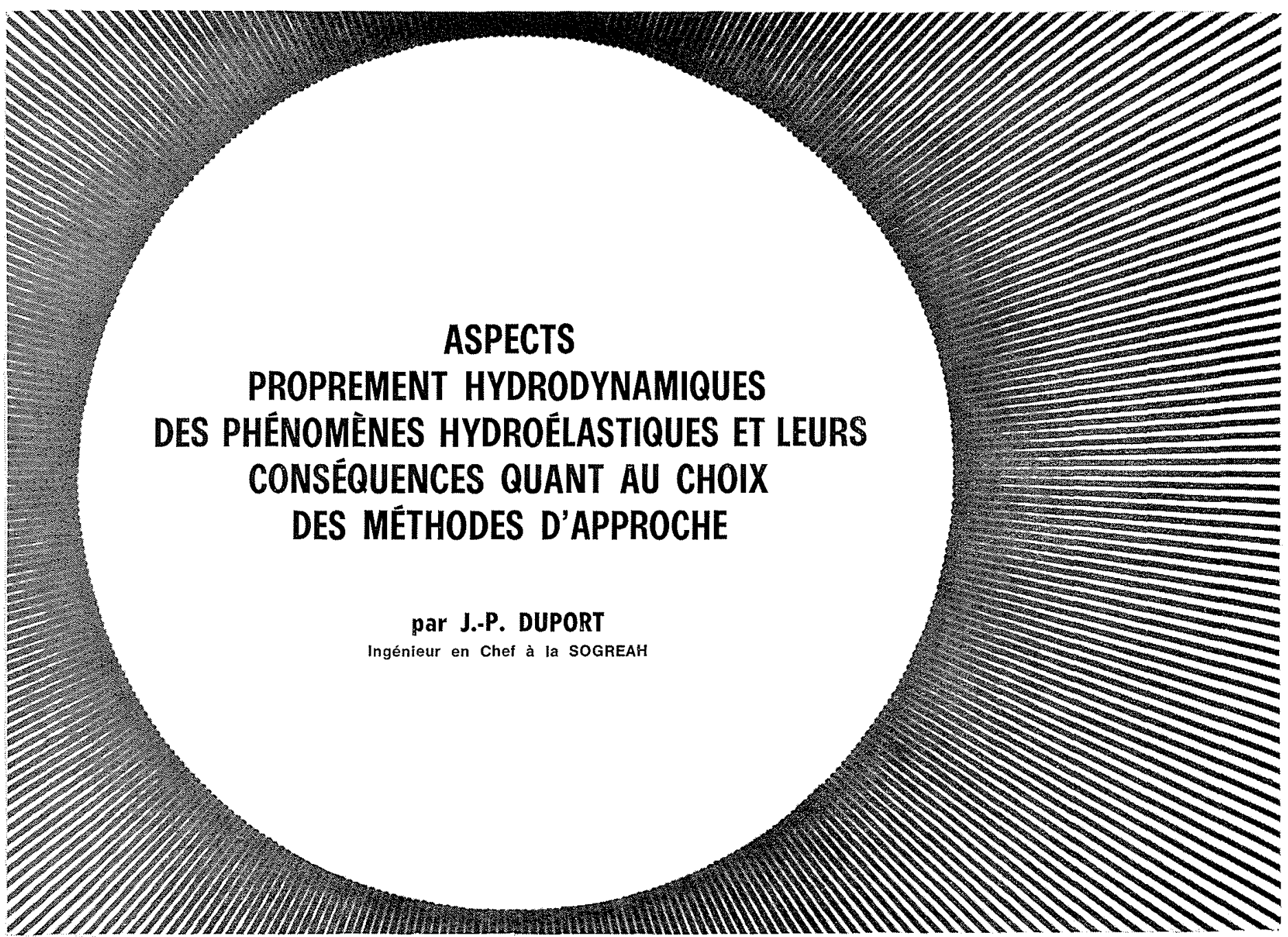

Hydro et aéroélasticité

La plupart des auteurs qui traitent d'aéro ou d'hydroélasticité définissent ces disciplines comme concernant les phénomènes impliquant des interactions mutuelles entre forces d'inertie, forces hydrodynamiques et forces élastiques.

S.R. Heller Jr (*) décrit le domaine de l'hydroélasticité en présentant tout d'abord les « diagrammes triangulaires 》 proposés en 1937 et 1946 par Collar $\left(^{* *}\right)$ comme description synthétique de l'aéroélasticité.

Ce sont ces triangles que nous reproduisons à la figure 1 où les lettres ont les significations suivantes :

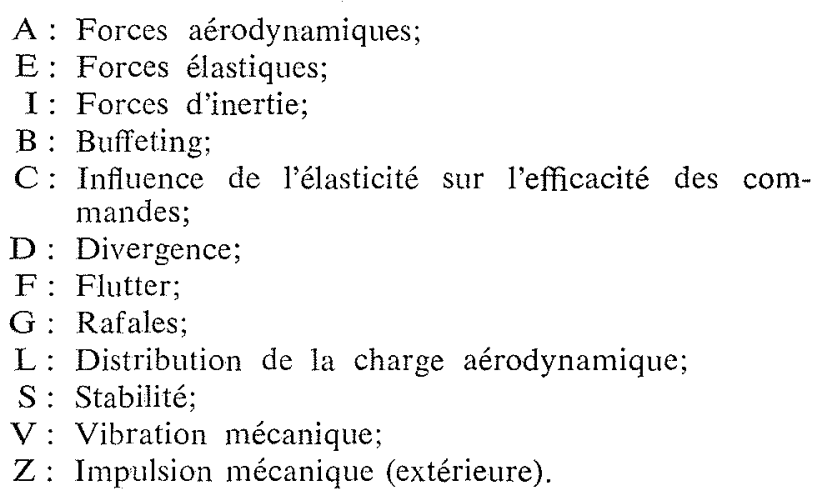

(\%) "Hydroelasticity " dans Advances in Hydroscience, vol 1-1964, Academic Press, New York et Londres.

(*) Collar A.R., Journal Royal Aeron. Sc, $\mathrm{n}^{\circ}$ 428, 1946
Heller propose ensuite un diagramme équivalent appli. cable au domaine de l'hydrodynamique navale. C'est celui que nous reproduisons en figure 2 où les sigles nouveaux par rapport aux précédents ont les définitions suivantes:

$\mathrm{H}$ : désigne les forces hydrodynamiques;

DR: signifie « réponse dynamique », l'auteur voulant dire par là réponse dynamique aux fluctuations de pression ayant pour origine la cavitation, la turbulence ou les instabilités de sillage;

$\mathrm{XR}$ : signifie la réponse dynamique aux explosions sous marines.

Heller présente enfin le «triangle 1980 » où figurent, en plus des précédentes:

IR: les « interactions de surface » c'est-à-dire : chocs sur les vagues, réentrée dans l'eau (hydroptères), slamming, etc.;

TM : les questions d'ancrage et de remorquage où intervient l'hydroélasticité (forces hydrodynamiques, élastiques, d'inertie) des câbles.

L'idée directrice de ces diagrammes est intéressante, car elle illustre les effets des interactions entre deux de ces forces ou entre les trois. Nous craignons cependant que cette image présente une vue trop simplifiée des choses.

En particulier, on ne voit pas figurer parmi les forces, les forces de champ (gravité en particulier) et cette lacune serait gênante si l'on voulait utiliser ces diagrammes pour une analyse des problèmes de similitude.

Le classement des problèmes hydroélastiques par Heller ne concerne d'ailleurs que "l'hydrodynamique navale 》 qui est en quelque sorte le domaine de «l'hydrodynamique extérieure ». Pour compléter le tableau de l'hydro- 


\section{J.P. DUPORT}

élasticité, il faudrait y ajouter tout ce qui concerne «l'hydrodynamique interne » c'est-à-dire celle des circuits en charge et des machines, et enfin un troisième domaine (qui n'a pas son correspondant en aérodynamique): celui des écoulements à surface libre qui se distingue de l'hydrodynamique extérieure à surface libre par le fait de sections transversales limitées.

Les considérations qui précèdent mettent en lumière certains des caractères qui distinguent les problèmes d'hydroélasticité des problèmes d'aéroélasticité. Cette distinction est certainement d'une assez grande importance pratique, ne serait-ce que pour bien saisir en quoi les résultats et les méthodes de l'aéroélasticité (développée bien avant l'hydroélasticité) doivent être amenclés où complétés pour être appliqués à l'hydroélasticité.

Une première différence, importante, tient aux propriétés mécaniques des fluides concernés: masse volumique et compressibilité.

La différence de densité entre les liquides et les gaz " usulels » se traduit par des ordres de grandeur très différents du « rapport de masse » en hydro et en aéroélasticité. Cela est suffisamment connu pour qu'il soit inutile d'y insister.

La densité beaucoup plus élevée des liquides est également à l'origine du gradient de pression hydrostatique dont l'incidence sur les déformations (et éventuellement la stabilité) élastiques peut être appréciable, ce qui n'est évidemment pas le cas en aéroélasticité.

La faible compressibilité des liquides a pour effet que la célérité des ondes de pression y est notablement plus élevée que dans les gaz et cette différence est encore accentuée par le fait que les vitesses les plus élevées auxquelles s'intéressent l'hydrodynamique sont beaucoup moins élevées qu'en aérodynamique (les avions les plus rapides ont une vitesse plus de 10 fois supérieure à celle des véhicules nautiques les plus rapides).

Il en résulte que l'hydrodynamique ne s'intéresse pratiquement - en régime permanent - qu'au domaine subsonique et même incompressible (il existe des exemples, à notre connaissance fort peu nombreux, de problèmes d' « hydrodynamique supersonique »: entrée dans l'eau de projectiles ultra-rapides, jets d'eau supersoniques).

En régime instationnaire - et la plupart des problèmes d'hydroélasticité ont un caractère instationnaire - la compressibilité du fluide peut cesser d'être négligeable. Il en est évidemment de même en aéroélasticité. Cependant la compressibilité plus faible des liquides a pour conséquence que l'hypothèse du fluide incompressible a un champ d'application plus étendu qu'en aéroélasticité.

Une seconde distinction entre hydro et aéroélasticité résulte de la présence de surfaces libres dans un grand nombre de problèmes hydrodynamiques, ce qui n'a évidemment pas d'équivalent en aérodynamique.

La surface libre est susceptible d'affecter de façon déterminante l'interaction entre les déformations élastiques des parois et le fluide; on peut citer par exemple à ce sujet l'émission d'un système de vagues sous l'effet de vibrations.

Par ailleurs, les phénomènes de houle qui affectent la surface libre donnent lieu à toute une classe de problèmes proprement hydroélastiques (réponse des structures aux sollicitations dues à la houle).

Enfin la surface libre représente une discontinuité de densité qui peut être à l'origine de brusques variations des forces hydrodynamiques affectant les structures (choc à l'émersion et surtout à l'immersion) et par suite de problèmes hydroélastiques qui n'ont évidemment pas d'équivalents en aéroélasticité.
Il y a enfin un autre domaine propre à l'hydroélasticité et qui ne peut être traité par une simple extension des modèles de l'aéroélasticité : c'est celui des écoulements cavitants. Nous avons en vue ici non seulement la prise en compte du phénomène de cavitation dans le modèle d'écoulement, mais aussi linteraction assez fréquente entre les déformations élastiques et le processus de cavitation.

\section{Essai de classement des problèmes hydroélastiques}

\section{Forces affectani les structures.}

Si l'on convient de définir l'hydroélasticité comme la discipline traitant des interactions mutuelles entre les forces hydrodynamiques, les réactions élastiques et les forces d'inertie, il faut reconnaitre alors qu'elle ne recouvre pas l'ensemble des problèmes qui résultent de l'inévitable élasticité des structures solides soumises à l'action des écoulements.

Les forces extérieures appliquées au solide, et qui y font naître réactions élastiques et déformations, ne se limitent pas, en effet, aux seules forces hydrodynamiques de pression et/ou de frottement fluide ayant pour origine l'écoulement.

Les structures sont également sollicitées par le liquide par l'intermédiaire

- de la pression statique (stationnaire, résultant par exemple de la pressurisation des écoulements clos);

- des gradients de pression dus aux forces de champs (gravité, forces de champs en écoulement relatif) (*);

- des fluctuations de pression d'origine indépendante de l'écoulement (par exemple du fait d'une source acoustique)

Les déformations nées de ces forces autres qu'hydrodynamiques sont à prendre en compte, au même titre que les forces hydrodynamiques, dans l'analyse et la prévision du comportement des systèmes comportant des structures solides partiellement ou totalement immergées dans un liquide.

Citons par exemple le problème de l'instabilité d'immersion des corps totalement immergés, instabilité qui s'explique par le seul jeu des forces hydrostatiques et des forces élastiques en l'absence de toutes force hydrodynamique.

Les effets des forces hydrauliques autres qu'hydrodynamiques peuvent en général être étudiés séparément du problème proprement hydroélastique. Il ne faut cependant pas perdre de vue que les déformations et déplacements élastiques résultent de l'ensemble de ces forces et pas seulement d'une partie d'entre elles. Cette séparation peut n'être pas licite, par exemple en ce qui concerne les pressions sonores : dans certains cas, il y a réaction de la perturbation sonore sur l'écoulement: par exemple par le développement de zônes cavitantes, et peut-être même par une influence sur la transition laminaire/turbulent.

\section{Effets hydroélastiques.}

Revenant maintenant au domaine propre de l'hydroélasticité (où sont présentes les forces élastiques et les forces hydrodynamiques), la première question qui se pose pour chaque problème particulier, n'est-elle pas de savoir si l'on doit recourir à un modèle hydroélastique et auquel.

(*) Il ne faut pas oublier que les forces de champ agissent également directement sur les masses propres des structures. 


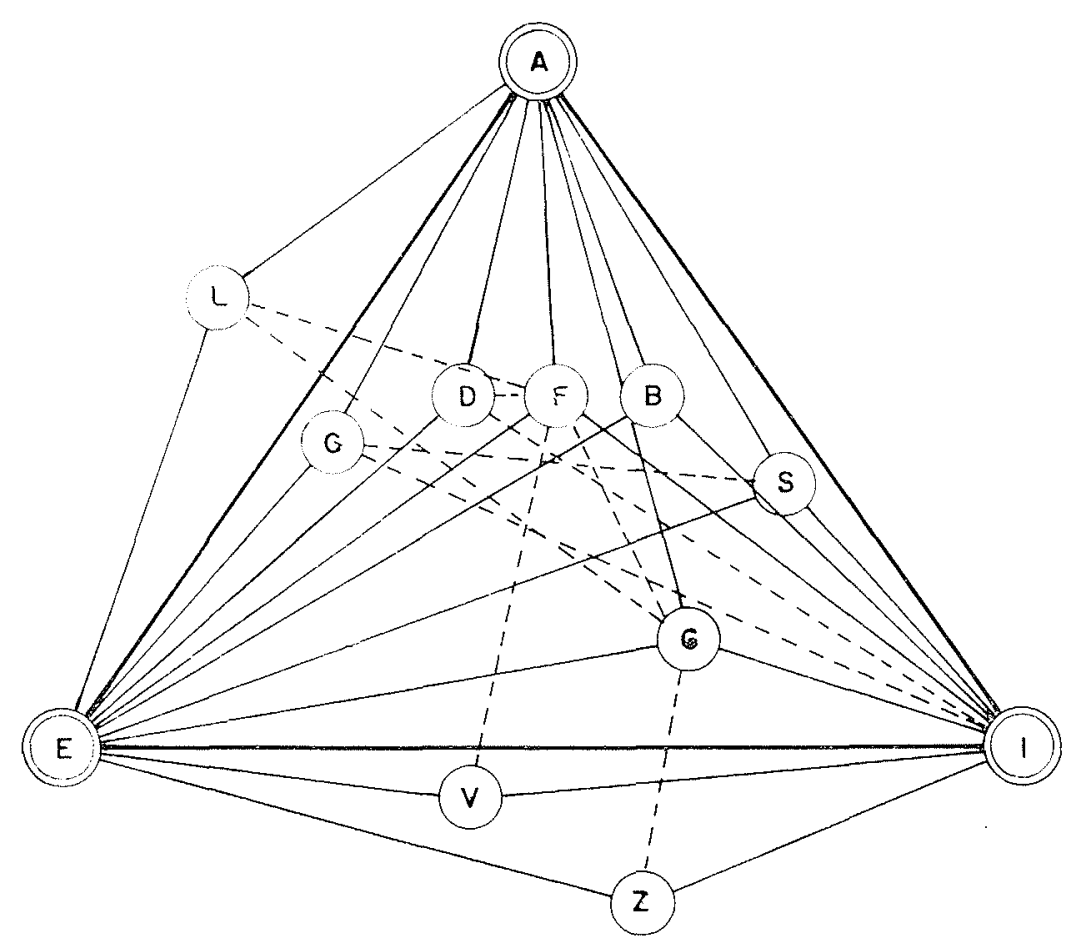

$1 /$ Aéroélasticité. D’après Collar cité par S.R. Heller

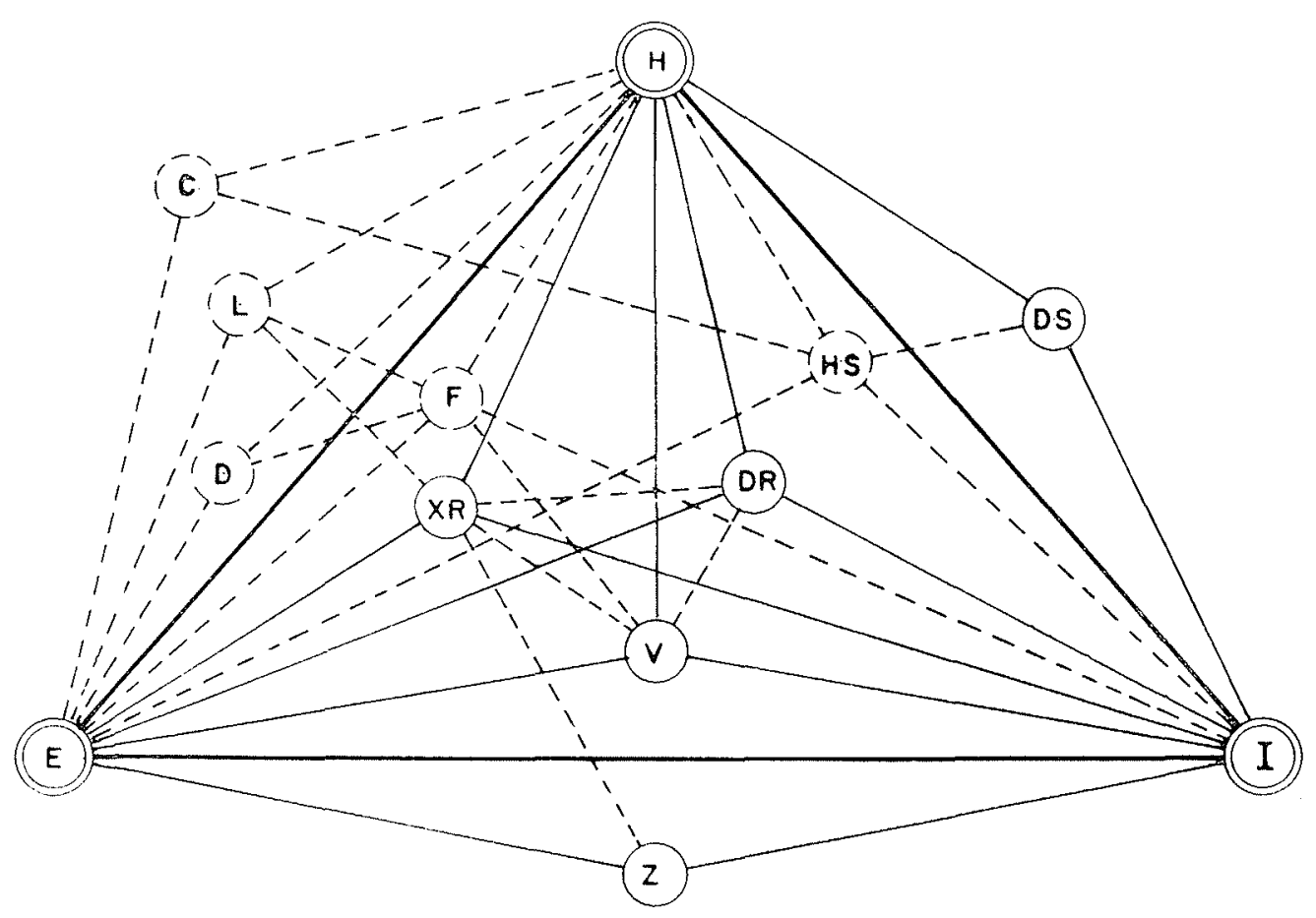

2/ Hydroélasticité. Daprès S.R. Heller 
En fait, aucun corps n'étant infiniment rigide, la pression hydrodynamique engendre nécessairement toujours déformation et/ou déplacement des parois en contact avec le liquide, ces déformations géométriques réagissant à leur tour sur l'écoulement, mais leur effet peut ne pas dépasser un seuil tel que l'analyse de l'écoulement faite dans l'hypothèse de la parfaite rigidité reste valable. On conçoit bien que ce seuil peut différer suivant la grandeur en cause et suivant le but de l'analyse.

\section{ECOULEMENTS STATIONNAIRES.}

Pour préciser cette notion des « effets hydroélastiques», examinons tout d'abord le cas d'écoulements stationnaires par rapport à un référentiel lié à la structure solide. Par stationnaires, nous entendons que les conditions aux limites sont stationnaires - y compris la position de la structure si elle était rigide - les seules instationnarités étant celles de la turbulence.

L'élasticité (et éventuellement la plasticité) des structures peut se manifester :

1. Par des déformations et déplacements stationnaires et limités des structures, n'entrainant que des modifications relativement faibles des pressions et des forces, et sans effet sur la nature de l'écoulement. (Par nature de l'écoulement, nous entendons par exemple: cavitant ou subcavitant, laminaire ou turbulent, décollé ou sain, etc.).

Ce cas est celui où l'hypothèse de rigidité est applicable (sous réserve tout au plus de la prise en compte d'éventuelles corrections marginales si l'on recherche une extrême précision).

2. Par des modifications géométriques stationnaires faibles des structures, mais ayant une incidence apparemment disproportionnée sur les forces hydrodynamiques et/ou sur la nature de l'écoulement.

Un exemple très typique est celui des profils porteurs et plus généralement des écoulements à connectivité multiple : la portance (et le moment de portance) d'une aile peut être modifiée de façon significative par la variation d'incidence (ou de cambrure) résultant des flèches de l'aile ou de son support.

Du fait que les déformations locales sont faibles, les modifications des vitesses et pressions locales sont faibles, mais la portance résulte non pas des pressions locales mais des différences entre les pressions locales sur chaque face. Cette différence de pression est elle-même liée à la circulation autour du profil qui varie relativement rapidement avec l'incidence (condition de Kutta).

On ne trouve rien d'équivalent si on s'intéresse à l'écoulement confiné dont les parois seraient soumises à des déformations élastiques comparables à celle du profil précédent; d'une part les pressions auxquelles on s'intéresse ici varieront peu, et d'autre part, même les différences de pression entre paroi inférieure et supérieure varieront moins, l'écoulement étant beaucoup plus guidé en amont et en aval de la perturbation.

Un autre exemple possible d'influence hydrodynamique notable de déformations élastiques limitées peut être apporté par l'exaltation des traînées de frottement sous l'effet des ondulations de surface provoquées par la charge hydrodynamique.

Il existe également des cas où des déformations stationnaires faibles peuvent engendrer des effets apparemment peu progressifs; ceci peut apparaître quand, dans la zone concernée, les conđitions d'écoulement sont proches d'un seuil de transition :

- transition entre couche limite laminaire et couche turbulente;
- décollement (on sait qu'une très faible déformation ou aspérité peut dans certains cas déclencher un décollement dans un écoulement retardé);

- seuil de cavitation.

Les exemples qui précèdent montrent bien que les problèmes « d'hydroélasticité stationnaire » ne se limitent pas à ceux de la divergence dont nous allons parler maintenant.

3. Par des déformations stationnaires mais non limitées (divergence).

On peut dire, en simplifiant un peu, que la divergence résulte du fait que l'équilibre entre forces hydrodynamiques et forces élastiques n'est pas stable en raison du fait que la déformation engendre une croissance des forces hydrodynamiques plus rapide que la croissance de la force élastique antagoniste.

Il est bien évident que la divergence affecte de façon notable l'écoulement et toutes les grandeurs qui y sont liées. La nature de l'écoulement (couches laminaires ou turbulentes, décollements, cavitation, etc.) peut s'en trouver entièrement modifiée, et il y a lieu d'en tenir compte surtout si l'on s'intéresse à l'effet de la divergence et non pas seulement aux conditions limites de son apparition.

\section{Par des déformations instationnaires (vibrations).}

Puisque nous examinons ici le cas des écoulements stationnaires (c'est-à-dire dont le seul défaut éventuel de stationnarité est la présence de turbulence), les vibrations dont il est question ici proviennent:

- soit de la réponse des parois à la turbulence (bruit d'écoulement);

- soit d'une « auto-excitation».

L'exemple le plus fréquemment cité d'auto-excitation affectant un écoulement «stationnaire » est le flutter. Il semble bien que, sous les réserves indiquées précédemment, les modèles et les méthodes de l'aéroélasticité soient transposables ici à l'hydroélasticité.

Nous voudrions citer deux autres exemples de phénomènes d'auto-excitation parfois rencontrés en hydraulique (et vraisemblablement aussi en aéraulique) :

a) Résonance sur un conduit long. C'est ce qui se produit par exemple lorsqu'une vanne est placée sur une conduite et que les déformations élastiques de son système d'accrochage, ou de son étanchéité peuvent engendrer des variations de débit. Dans certaines circonstances, il peut y avoir résonance entre la vibration de la vanne et la période propre d'oscillation de pression de la conduite.

b) Effet de clapet battant.

Le phénomène de clapet battant est provoqué par un laminage de l'écoulement entre une paroi fixe et un organe mobile soumis à une réaction hydraulique qui s'inverse lorsque le mobile est très rapproché de la paroi. Il s'agit d'un effet hydroélastique lorsque le mobile est lié élastiquement aux structures fixes.

En raison de l'amortissement dû aux frottements fluides et de l'amortissement interne des structures, l'existence d'oscillations entretenues dans les exemples qui précèdent ne peut à notre avis s'expliquer que par la présence de non linéarités et de relaxations affectant soit le phénomène hydraulique (régime de décollement par exemple) soit les frottements des structures. Il y a évidemment à tenir compte de ces remarques dans le choix des modèles.

\section{ECOULEMENTS INSTATIONNAIRES.}

Nous aborderons maintenant le cas des écoulements instationnaires, et il nous paraît intéressant de distinguer deux origines possibles de l'instationnarité : les instationnarités 
« spontanées » des écoulements et celles qui résultent directement du caractère non stationnaire des conditions aux limites.

Il est bien connu que des instabilités d'écoulement peuvent prendre naissance alors même que les conditions aux limites sont stationnaires. (Cette question a été largement abordée au cours des $8^{\circ}$ Journées de l'Hydraulique de la SHF en 1964 et dont le sujet était précisément: « Les instabilités en hydraulique et en mécanique des fluides ».

Certaines de ces instabilités se rencontrent aussi bien en aérodynamique qu'en hydrodynamique; citons par exemple :

- les instabilités de confluences, en particulier les sillages avec tourbillons de Karman;

- les décollements instables (instabilité du point de décollement et lâcher intermittent de tourbillons depuis la zone décollée);

- les fluctuations du point de transition entre régime laminaire et régime turbulent de la couche limite.

D'autres instabilités n'apparaissent que dans les écoulements de liquide; par exemple:

- celles qui sont provoquées par une aération intermittente de l'écoulement;

- les oscillations des surfaces libres;

- les instabilités propres aux écoulements affectés par la cavitation :

- poche de cavitation fluctuante avec jet rentrant intermittent;

- collapsus de cavité, ou encore collapsus « cohérent» d'une zone émulsionnée par cavitation.

Ces instablités «spontanées » prennent naissance même en présence de parois rigides et on ne peut les assimiler aux instabilités par « auto-excitation » dont il est question plus tôt. On peut même dans certains cas séparer l'analyse de ces instabilités et la détermination de la réponse des structures. Toutefois, il est souvent difficile de statuer a priori sur l'existence ou l'absence de réaction des vibrations de structure sur les instabilités de l'écoulement: la fréquence des lachers de tourbillons, ou des collapsus intermittents de cavitation par exemple est certainement fort souvent influencée directement par les vibrations des parois.

Les écoulements proprement instationnaires sont ceux qui résultent de conditions aux limites instationnaires; il leur correspond des probièmes hydroélastiques concernant la réponse des structures aux forces hydrodynamiques instationnaires.

Le cas des structures fixes ou mobiles soumises à la houle en est un exemple, et nous avons vu qu'il s'agit d'un problème qui ne peut se traiter par des modèles transposés de l'aéroélasticité, en raison, entre autres, de la présence de la surface libre, de l'incidence du gradient de pression hydrostatique et de la possibilité de chocs sur la surface libre.

Les machines tournantes constituent un domaine impor tant de l'hydrólasticité, dans lequel il existe une instationnarité cyclique liée au déflement des rotors et des stators; la même remarque s'applique aux hélices de navires en raison de l'effet de sillage de la carène et de l'intéraction hydrodynamique de la carène et de l'hélice. Certains modèles de l'aéroélasticité sont, sous les réserves formulées au début, en grande partie transposables à cette classe de problèmes hydroélastiques.

La même remarque s'applique à l'étude hydroélastique de transitoires résultant du mouvement commandé d'organes de circuit (vanne par exemple).

Enfin nous avons déjà signalé l'existence de transitoires propres au domaine de l'hydrodynamique: ceux qui concernent le mouvement de mobiles traversant la surface libre (émersion ou immersion), avec choc possible. Le développement de modèles propres à l'hydroélasticité est évidemment nécessaire pour traiter de tels problèmes.

\section{Quelques réflexions sur les méthodes d'approche de l'hydroélasticité}

Une première série de remarques s'applique aussi bien à l'aéroélasticité qu'à l'hydroélasticité et concerne les hypothèses que l'on est amené à faire au sujet des propriétés du fluide et des caractères de l'écoulement:

\section{Fluide parfatt ou fluide réel.}

Il peut exister des cas où des modèles en fluide parfait conviennent à lapproche au moins approximative d'un problème d'écoulement en parois rigides, mais ne permettent pas d'aborder de façon licite les aspects hydroélastiques de ce même écoulement. C'est ce qui se passe par exemple lorsque des déformations ou des vibrations hydroélastiques et des évolutions de couches limites (point de transition, décollement) s'influencent mutuellement (*). C'est également le cas des écoulements stationnaires, en moyenne sujets à des instabilités qui restent limitées en l'absence de résonance hydroélastique.

Fluide compressible ou inCompressible.

Nous avons fait allusion précédemment au fait que des écoulements stationnaires du domaine "incompressible » peuvent être le siège de perturbations hydroélastiques instationnaires nécessitant le recours à des modèles en fluide compressible. Des critères simples applicables à un grand nombre de cas usuels devraient pouvoir être établis à ce sujet.

\section{ECOULEMENTS A CONNECTIVITÉ MULTIPLE.}

L'indétermination des solutions en fluide parfait de ce type d'écoulement est levée, en régime permanent, par l'application de la condition de Kutta. L'hydroélasticité instationnaire (et plus généralement l'hydrodynamique instationnaire) pose le problème de l'extension de cette condition au domaine instationnaire. A notre connaissance cetle question n'a été qu'imparfaitement explorée, tout au moins dans le domaine propre à l'hydroélasticité.

D'autres remarques concernent spécialement l'hydroélasticité :

- Nous avons noté précédemment que la présence de la surface libre implique le recours à des modèles non disponibles évidemment en aéroélasticité. La même remarque s'applique à la présence d'un fort gradient de pression hydrostatique dans les liquides.

Dans les cas où la nature des problèmes conduit à recourir à des «modèles hydroélastiques concrets » combinant modèle hydraulique à surface libre et modèle de structure, l'application de la similitude de Froude engendre souvent des difficultés pratiques pour le modèle de structure. A cette difficulté s'ajoute, par exemple dans le cas de chocs de réentrée, le problème de la compressibilité de l'eau (et également de l'air entraîné). Ces difficultés ne peuvent être levées que par une étude préalable de qualification de si-

(*) On pourrait également remarquer que les modèles en fluide parfait négligent l'amortissement visqueux et turbulent des vibrations élastiques, mais cet effet peut souvent être introduit comme teme correctif à partir d'un modèle en fluide parfait. 
militude qui ne nous semble pas pouvoir, dans l'état actuel des connaissances, procéder de l'application de critères généraux préétablis.

- On ne saurait trop insister à notre avis sur le risque de couplage entre le phénomène de cavitation et les vibrations hydroélastiques:

- d'une part la cavitation est par elle-même une source d'instabilité hydrodynamique pouvant se coupler avec les structures;

- d'autre part, elle engendre des discontinuités ou des nonlinéarités des forces hydrodynamiques, pouvant être à l'origine de flutter non prévisible en écoulement non cavitant. (Analogie avec le stall-flutter.)

\section{Conclusion}

Le bref survol des problèmes de l'hydroélasticité n'a pas d'autre ambition que de proposer un classement des problèmes en fonction des circonstances hydrodynamiques. Nous pensions également utile d'insister sur les domaines de l'hydroélasticité qui ne paraissent pas pouvoir être abordés par une simple extension des méthodes de l'aéroélasticité.

Enfin nous voudrions terminer en insistant sur l'intérêt qui s'attache à développer des méthodes d'approche de plus en plus représentatives et de plus en plus sûres en hydroélasticité. On peut escompter du progrès de l'hydroélasticité une réduction des coûts et des durées des opérations de développement dans un vaste domaine de la technique.

Les domaines où l'hydroélasticité joue déjà et jouera de plus en plus un rôle important sont principalement ceux où les progrès de la technique et les nécessités de l'économie conduisent aux réalisations géantes et ceux qui impliquent des écoulements très rapides. On peut citer par exemple (mais cette liste est évidemment très incomplète) :

- les conduites forcées et surtout les grandes vannes (qui à notre connaissance ont été, il y a déjà bien des années, l'objet des premières études hydroélastiques);

- les machines hydrauliques géantes (turbines et turbines pompes) et les hélices de navire;

- les réacteurs nucléaires rapides refroidis en sodium;

- les navires géants;

- les grandes plateformes flottantes ou fixes mises en auvre en océanologie et plus spécialement dans l'exploitation pétrolière off-shore;

- les navires et engins nautiques rapides où l'acuité des problèmes hydroélastiques est accentuée par l'incidence des impératifs de légèreté. On peut rapprocher de cette catégorie de «sujets d'hydroélasticité » le problème de l'amerrissage des avions.

L'importance de ces sujets justifierait à elle seule l'intérêt qui se manifeste actuellement pour l'hydroélasticité et pour le développement de ses méthodes d'approche.

\section{Discussion}

Merci M. DuporT, dit M. Ie Président; vous avez manifesté quelques petites différences d'opinion avec M. le Professeur FortirR. Celui-ci veut-il intervenir?

J'ai longuement développé dans ma communication écrite, répond M. Fortier, les questions de terminologie. Historiquement, les premiers problèmes du domaine qui nous occupe ont été les problèmes d'aéroélasticité. Dans ces problèmes, l'écoulement est essentiellement «instationnaire » c'est pourquoi j'ai conservé ce caractère dans ma définition. Toutefois, il est certain que les problèmes d'hydroélasticité «stationnaire» sont souvent importants; mais il faudrait, à mon avis, introduire, pour les désigner, un terme différent. Par ailleurs, je n'ai pas fait figurer dans ma définition le mot «élastique» car dans bien des cas les forces correspondantes n'interviennent pas.

D'autre part, j'ai bien insisté sur le fait que l'onsquion se trouve dans le domaine du nombre de Reynolds critique, un très petit mouvement de la structure peut provoquer une grande variation des coefficients de traînée.

M. Canavelis souligne l'intérêt pratique du problème des vibrations des tuyauteries (comportant souvent des coudes non coplanaires) parcourues par des écoulements. Peut-on considérer dans un tel cas qu'il y a interaction entre la paroi et l'écoulement interne ? En tous cas, les phénomènes de résonances amplifient fortement les vibrations.

11 paraît improbable, répond $M$. Duport - sauf s'il s'agit de tuyaux souples - que l'on se trouve ici en présence d'une interaction des déformations élastiques de la paroi sur l'écoulement local bien que les courants secondaires dans les coudes puissent créer des instabilités hydrodynamiques de grande amplitude génératrices de coups de bélier, phénomène dans lequel existe effectivement une interaction des déformations élastiques et de l'écoulement.

Par contre, nous avons rencontré dans des essais de coude à ailettes (qui réduisent notablement les instabilités susvisées) des phénomènes de couplage entre l'écoulement et les vibrations mécaniques des ailettes; nous avons constaté que les «lachers» de tourbillons sont fortement influencés par les vibrations élastiques des ailettes.

\section{Wegner pose la question suivante}

M. Duport a beaucoup parlé du problème de la ré-entrée de véhicules spatiaux ou marins. A ce sujet, j'aimerais savoir si l'ablation des boucliers thermiques est gouvernée uniquement par des données thermodynamiques ou si les effets aéroélastiques jouent un rôle important?

Cette question, répond $M$. le Président, fait encore l'objet de recherches. Pour le moment, on se contente de vérifier en soufflerie à haute température que le bouclier et les produits qui le constituent résistent; on ne constate pas de phénomènes instationnaires très importants.

A ce propos, on peut évoquer l'effet POGO qui résulte du couplage entre les déformations d'une structure extrêmement légère et les mouvements de la masse de liquide avec surface libre qu'elle contient. Les engins spatiaux emportent un poids de liquides qui peut atteindre $95 \%$ de leur poids total; l'écoulement de ces liquides à travers les pompes et les canalisations, compte tenu des accélérations propres du véhicule spatial, posent des problèmes hydroélastiques très difficiles que les méthodes mathématiques de $M$. DAT ne suffisent pas à calculer; il faut recourir à l'expérience.

A la suite d'un échange de vues entre M. DUPORr - qui a étudié l'effet POGO en cryogénie - et M. le Président, M. DAT précise :

L'effet POGO s'explique par le couplage entre les modes propres de vibrations longitudinaux des engins et la propulsion. L'O.N.E.R.A. a entrepris une recherche générale sur ce phénomène, et la Direction des Structures étudie les méthodes de calcul des modes de vibrations longitudinaux en présence du combustible liquide. Il faut prévoir le comportement de ce combustible, et notamment les fluctuations de la pression dans les fonds de réservoirs. On essaie actuellement d'étendre la schématisation par éléments finis au fluide compressible.

M. le Président lève la séance en remerciant les conférenciers, les auteurs de mémoires et les personnes qui ont bien voulu prendre part à la discussion. 